\title{
ORNITHOBACTERIUM RHINOTRACHEALE (ORT) INFECTION IN RABBITS
}

Moshira, A. El-Abasy '); Amal, E. Youssif ${ }^{2)}$; Sohair, A. Attia ${ }^{3)}$ and El-Gohary, A. $A^{1)}$

1) Poultry and Rabbit Diseases Department, Faculty of Veterinary Medicine Kafrelsheikh University.

2) Animal Health Institute, Dokki, Giza.

3) Doctorate of Veterinary Medicine, Kafrelsheikh University.

\section{ABESTRACT}

This work was aimed to investigate the epidemiology and possible ways of diagnosis, treatment and control of Ornithobacterium rhinotracheale (ORT) as an emerging respiratory pathogen in rabbits during the period from 2013-2015. For this purpose, bacteriological, biochemical, serological and histopathological examinations for the collected samples from suspected diseased 300 rabbits at different districts in Kafrelshiekh Governorate suffered from respiratory signs with nasal Discharge, depression with ruffled fur, decreased food consumption with poor performance and expectoration of blood stained mucus just prior to death. The results revealed isolation of 21 isolates with an incidence of $7 \%$. The isolates were identified morphologically and biochemically by API 20 NE strip. Serological identification of the isolated strains using agar gel precipitation test revealed that all isolated ORT strains were belonging to serotype A. Antibiogramme of the isolated ORT strains revealed sensitivity to sulphamethoxozole + trimethoprine, spiramycin, neomycin, ampicillin, amoxicillin, ciprofloxacin and tetracycline but resistant to penicillin, streptomycin, clindamycin, lincomycin, gentamycin, vancomycin and 
colistin sulphate.

Experimental infection of 3-month-old rabbits with isolated ORT strains and treatment trials using sulpha-trimethoprine and coconut oil were carried out. Clinical signs, post-mortem lesions and mortality with re-isolation of the infected strains were studied in details. Histopathological examination of different organs from experimentally infected rabbits revealed less severe lesions after treatment with sulpha-trimethoprine and coconut oil, in comparison with naturally infected rabbits.

\section{INTRODUCTION}

Commercial rabbit enterprises is an important industry for meat, fur and leather production. Respiratory infections are the most serious group of diseases affecting rabbits and accompanied by heavy economic loss due to increased mortality, increased condemnation rates, reduced fertility and medication costs. Several pathogens are indicated as possible causes of respiratory disorders either alone or in synergy with other micro-organisms.

The severity of clinical signs, duration of the disease, and mortality of Ornithobacterium rhinotracheale (ORT) outbreaks are extremely variable. They can be influenced by strain virulence, environmental factors, the immune status of the host, and the presence of other infectious agent (Van Empel et al., 1999).

ORT is a Gram-negative non-motile pleomorphic rod shaped bacterium which has been isolated from domestic birds with respiratory problems in Europe, South Africa, Egypt, Israel and USA, ORT has been 
isolated from chickens (Vanda mme et al., 199; El-Gohary,1998; and Roussan et al 2011) and turkey (Hafez, 1996).

The organism has been isolated from rabbits suffered from respiratory disorder in Egypt (Attia, 2008), ORT has been reported to be associated with clinicopathological effects in Muscovy and Balady ducks (El-Abasy 2008).

ORT is difficult to be differentiated from other respiratory pathogens affecting rabbits specially Pasteurella multocida infections duo to colonies of ORT on culture may resemble those of Pasteurella multocida on routine isolation media and cause confusion in confirming.

ORT infection can be successfully treated with antibiotic, but the bacterium rapidly developed antibiotic resistance (Devriese et al., 2001). So the aim of this work was to investigate the incidence. isolation and identification of ORT and treatment trials of ORT experimentally infected rabbits.

\section{MATERIALS AND METHODS}

\section{Collected Samples:}

Samples have been collected from 40 rabbit farms distributed in different localities in Kafrelshekh Governorate suffering from respiratory manifestation were examined for presence of ORT during the period from 2013 to 2015. Samples were taken under aseptic condition in ice tanks to the laboratory. The history of investigated rabbit farms, clinical signs, postmortem lesions as well as mortality rate were described in Table (1).

\section{Bacterial Isolation:}


Under aseptic condition loopfuls from (sinus, trachea, lung, and liver) of diseased rabbits were inoculated in brain heart infusion broth and incubated at $37^{\circ} \mathrm{C}$ for 24 hours. A loopful from broth into $10 \%$ sheep blood agar media containing $10 \mu \mathrm{g}$ gentamicin sulphate/ $\mathrm{ml}$ blood agar media to inhibit the over growth of the other bacteria (Back et al.,1996) and incubated at $37^{\circ} \mathrm{C}$ for $24-48$ hours under $7.5 \% \mathrm{CO}_{2}$ tensions. Suspected ORT colonies, after purification were identified morphologically (shape, size, colour, appearance, odour, and elevation). Finally films were prepared from the suspected pure colonies stained with Gram's stain and examined microscopically (Chin and Charlton, 2000).

\section{Biochemical identification:}

Suspected colonies of ORT were inoculated into buffer peptone water at $37^{\circ} \mathrm{C}$ for 48 hours under $7.5 \mathrm{CO}_{2}$ tension. Using micropipette or syringe for inoculation of this culture into different tests of API $20 \mathrm{NE}$ strip, incubated at $30^{\circ} \mathrm{C}$ for 48 hours. The interpretation of the test was carried out according to the instruction of the kits producer (Hafez, 1996).

\section{Serological identification:}

Suspected ORT strains were serologically identified by usingagar gel precipitation test (AGPT) (Van Emple et al, 1999). Approximately 6 $\mathrm{ml}$ of sterile agar in $60 \mathrm{~mm}$ petri-dish was used to obtain $2.8 \mathrm{~mm}$ thickness. Patterns consisting of six or seven wells (3 $\mathrm{mm}$ in diameter) located around a central well at a distance of $5 \mathrm{~mm}$ were punched out the agar. The central well was filled with the antigen extract and the 
peripheral ones with the anti-sera against ORT organism. The plates were incubated at room temperature and evaluated after 24, 48 and 72 hours. The observation for perception lines occur under ultra violet light.

\section{Experimental infection:}

Thirty 3-month-old hybrid rabbits obtained from rabbit farm of Faculty of Agriculture Collage, Kafrelshickh University were used for pathogenicity test. Fed green clover and pellet ration, the food and water were added ad-libtum. Rabbits were divided into two groups each group contained 15 rabbits. Each group. divided into three subgroup of 5 rabbits each in separate box. The first group was infected by ORT serotype A by an aerosol in dose $1 \times 10^{9}$ C.F.U/ml, and the second group was kept uninfected kept under observation for 4 weeks.

\section{Antibiogramme:}

The antibiotic sensitivity test of the isolated ORT strains were investigated against 19 antimicrobial agents using disc diffusion technique. The colony of ORT was picked up and suspended in tryptose soy broth to a turbidity of $0.5 \mathrm{McF}$ arland scale. One hundred microliters of the culture was spread uniformly onto the different media:

- Muller Hinton agar supplemented with $10 \%$ sheep blood.

- $10 \%$ sheep blood agar

The discs were dispensed on the culture and the plates were incubated at $37^{\circ} \mathrm{C}$ for 24 hours under micro aerophilic condition .The inhibition zones were measured according to National Committee for Laboratory Studies (Back et al., 1997).

\section{Treatment Trials:}


Based on the result of the in vitro antibiogramme, sulphatrimethoprim (SXT) and coconut oil were used for treatment trials of ORT experimentally infected rabbits.

\section{Histopathological examination:}

Specimens of sinus, larynx, trachea, lung, heart, liver and kidney were taken from freshly dead and sacrificed experimentally infected rabbits, fixed in 10\% neutral buffer formalin (Lillie,1984), washed, dehydrated in different concentration of alcohol, cleared in xylol and embedded in paraffin. The paraffin embedded blocks were cut into $5 \mu \mathrm{m}$ section and stained with Haematoxylin and Eosin (H \&E) stain according to Bancraft and Gamble (2007).

\section{Statistical analysis:}

Statistical analysis of obtained data was carried out according to Petri and Watson (1999).

Table (1): History of investigated rabbit farms in Kafrelsheikh Governorate

\begin{tabular}{|c|c|c|c|c|c|c|c|}
\hline \multirow{2}{*}{$\begin{array}{c}\text { Farm. } \\
\text { No. }\end{array}$} & \multirow{2}{*}{ Distriets } & \multirow{2}{*}{$\begin{array}{c}\text { No. of } \\
\text { Rabbits in } \\
\text { Floek } \\
\end{array}$} & \multirow{2}{*}{ Breed } & \multirow{2}{*}{ Ctinital signs } & \multirow{2}{*}{ Postmortem Lesions } & \multicolumn{2}{|c|}{ Mortality rate } \\
\hline & & & & & & No. & $\%$ \\
\hline 1 & Kafrelsheikh & 600 & $\begin{array}{c}\text { New } \\
\text { Zealand }\end{array}$ & $\begin{array}{l}\text { Sneezing, cough and nasal } \\
\text { discharge }\end{array}$ & Trachitis and pneumonia & 40 & 6 \\
\hline 2 & Kafrelsheikh & 900 & $\begin{array}{l}\text { New } \\
\text { Zealand }\end{array}$ & Bloody mucoid discharge & Septicemia & 5 & 0.5 \\
\hline 3 & Kafrelsheikh & 500 & Dutch & Coughing and nasal discharge & $\begin{array}{l}\text { Sinusitis, trachitis and, } \\
\text { consolidation of lung }\end{array}$ & 5 & 1 \\
\hline 4 & Sakha & 1000 & Hybrid & $\begin{array}{l}\text { Mucoid nasal discharge and } \\
\text { conjunctivitis }\end{array}$ & $\begin{array}{l}\text { Congested lung and yoghourt } \\
\text { like exudates in the thoracic } \\
\text { cavity }\end{array}$ & 6 & 0.6 \\
\hline 5 & Sakha & 1000 & Hybrid & Dyspnea and poor growth & $\begin{array}{l}\begin{array}{l}\text { Trachitis and } \\
\text { pneumonia }\end{array} \\
\end{array}$ & 4 & 0.4 \\
\hline 6 & El-Reyad & 300 & $\begin{array}{c}\text { New } \\
\text { Zealand }\end{array}$ & Mucoid nasal discharge & $\begin{array}{l}\text { Trachitis, sinusitis and } \\
\text { haemorrhagic spots in lung }\end{array}$ & 10 & 3.3 \\
\hline 7 & El-Reyad & 500 & Bosuchate & $\begin{array}{l}\begin{array}{l}\text { Mild respiratory sings and } \\
\text { abortion }\end{array} \\
\end{array}$ & $\begin{array}{l}\text { Trachitis, sinusitis and } \\
\text { congested in uterus }\end{array}$ & 8 & 1.6 \\
\hline 8 & El-Hamol & 500 & Hybrid & Poor fur and respiratory sings & $\begin{array}{l}\text { Trachitis and haemorrhagic } \\
\text { spots in lung }\end{array}$ & 5 & 1.0 \\
\hline 9 & Kafrelsheikh & 600 & Hybrid & $\begin{array}{l}\text { Rales, nasal discharge and poor } \\
\text { weight }\end{array}$ & $\begin{array}{l}\text { Trachitis, pneumonia and } \\
\text { hydrothorax }\end{array}$ & 4 & 0.6 \\
\hline
\end{tabular}




\begin{tabular}{|c|c|c|c|c|c|c|c|}
\hline 10 & Kafrelsheikh & 500 & $\begin{array}{c}\text { New } \\
\text { Zealand }\end{array}$ & $\begin{array}{|lll|}\begin{array}{l}\text { Sneezing, cough } \\
\text { emaciation }\end{array} & \text { and } \\
\end{array}$ & $\begin{array}{l}\text { Trachitis, pneumonia and } \\
\text { hepatomegally }\end{array}$ & 3 & 0.6 \\
\hline 11 & Araymon & 800 & $\begin{array}{c}\text { New } \\
\text { Zealand }\end{array}$ & $\begin{array}{|lll|}\begin{array}{l}\text { Dyspnea } \\
\text { discharge }\end{array} & \text {,cough } & \text { and,nasal } \\
\end{array}$ & $\begin{array}{l}\text { Trachitis, pneumonia and } \\
\text { congestion of liver }\end{array}$ & 6 & 0.75 \\
\hline 12 & Dosque & 600 & Hybrid & $\begin{array}{l}\text { Rales, nasal discharge and } \\
\text { ocular discharge }\end{array}$ & $\begin{array}{lll}\text { Trachitis, } & \text { pneumonia } & \text { and } \\
\text { pyothorax } & & \end{array}$ & 3 & 0.5 \\
\hline 13 & Dosque & 500 & Bosuchate & 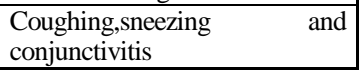 & $\begin{array}{l}\text { Trachitis, pneumonia and } \\
\text { hydrothorax }\end{array}$ & 5 & 1 \\
\hline 14 & Foa & 1000 & Hybrid & \begin{tabular}{|l}
$\begin{array}{l}\text { Mild respiratory signs and } \\
\text { salivation }\end{array}$ \\
\end{tabular} & $\begin{array}{l}\text { Slight congestion in trache and, } \\
\text { lung }\end{array}$ & 20 & 2 \\
\hline 15 & El-Hamol & 1000 & $\begin{array}{c}\text { New } \\
\text { Zealand }\end{array}$ & $\begin{array}{l}\text { Dyspnea, coughing and mucoid } \\
\text { nasal discharge }\end{array}$ & Trachitis and pneumonia & 5 & 0.5 \\
\hline 16 & Motobes & 500 & $\begin{array}{l}\text { New } \\
\text { Zealand }\end{array}$ & $\begin{array}{l}\text { Rales, coughing, ruffled fur, } \\
\text { ocular discharge and low body } \\
\text { weight }\end{array}$ & $\begin{array}{l}\text { Trachitis, pneumonia and } \\
\text { enlargement of liver }\end{array}$ & 10 & 2 \\
\hline 17 & Kafrelsheikh & 900 & $\begin{array}{l}\text { New } \\
\text { Zealand }\end{array}$ & $\begin{array}{l}\text { Coughing, rales and bloody } \\
\text { expectoration from mouth and } \\
\text { nose }\end{array}$ & $\begin{array}{l}\text { Septicemia and bloody } \\
\text { exudates in the thoracic and } \\
\text { abdominal cavity }\end{array}$ & 30 & 3.3 \\
\hline 18 & Sakha & 1000 & Hybrid & \begin{tabular}{|l} 
Sneezing, cough nasal \\
discharge and emaciation
\end{tabular} & Trachitis and pneumonia & 20 & 2 \\
\hline 19 & Elrhyad & 500 & Bosuchate & Rales, and ruffled fur & $\begin{array}{l}\text { Trachitis and small } \\
\text { hemorrhagic spots in lung }\end{array}$ & 3 & 0.6 \\
\hline 20 & Dosoque & 800 & $\begin{array}{c}\text { New } \\
\text { Zealand }\end{array}$ & Sneezing, rales and depression & Trachitisand pneumonia & 10 & 1.25 \\
\hline 21 & Sakha & 1000 & $\begin{array}{c}\text { Hybrid } \\
\text { boschute }\end{array}$ & \begin{tabular}{|l}
$\begin{array}{l}\text { Dyspnea, cough and nasal } \\
\text { discharge }\end{array}$ \\
\end{tabular} & $\begin{array}{lll}\begin{array}{l}\text { Trachitis } \\
\text { pneumonia }\end{array} & \text { and } & \text { unilateral } \\
\end{array}$ & 5 & 0.5 \\
\hline 22 & El-Reyad & 500 & Bosuchate & $\begin{array}{l}\text { Bloody mucoid, coughing and } \\
\text { nasal discharge }\end{array}$ & $\begin{array}{l}\text { Trachitis } \\
\begin{array}{l}\text { hydrothorax } \\
\text { consolidation of lung and } \\
\text { hepatomegaley }\end{array}\end{array}$ & 25 & 5 \\
\hline 23 & El-Reyad & 300 & $\begin{array}{c}\text { New } \\
\text { Zealand }\end{array}$ & Acular and nasal discharge & Conjunctivitis and trachitis & 15 & 5 \\
\hline 24 & Foa & 1000 & Hybrid & \begin{tabular}{|lll}
$\begin{array}{l}\text { Sneezing, depression and } \\
\text { abortion }\end{array}$ & \\
\end{tabular} & $\begin{array}{l}\text { Trachitis, pneumonia and } \\
\text { metritis }\end{array}$ & 7 & 0.7 \\
\hline 25 & Dosque & 500 & Bosuchate & \begin{tabular}{|lll} 
Depression, weakness and \\
ruffled fur
\end{tabular} & Trachitis and pneumonia & 3 & 0.6 \\
\hline 26 & Dosque & 600 & Hybrid & Dyspnea, coughing and rales & Trachitis and pneumonia & 4 & 0.6 \\
\hline 27 & El-Hamel & 1000 & $\begin{array}{c}\text { New } \\
\text { Zealand }\end{array}$ & $\begin{array}{l}\begin{array}{l}\text { Cough, sneeze and bloody } \\
\text { mucoid discharge }\end{array} \\
\end{array}$ & $\begin{array}{l}\text { Trachitis, bilateral pneumonia } \\
\text { and haemorrhagic septicemia }\end{array}$ & 20 & 2 \\
\hline 28 & Qelin & 1000 & Hybrid & $\begin{array}{l}\text { Respiratory sings and abortion } \\
\text { in breeder doe }\end{array}$ & $\begin{array}{l}\text { Trachitis, sever congestion in } \\
\text { lung, hepatomegally, metritis } \\
\text { and urinary bladder distention } \\
\text { with bloody urine }\end{array}$ & 50 & 5 \\
\hline 29 & Biala & 200 & $\begin{array}{c}\text { New } \\
\text { Zealand }\end{array}$ & Rales, sneezing and cough & $\begin{array}{l}\text { Sinusitis, trachitis and bilateral } \\
\text { pneumonia }\end{array}$ & 2 & 1 \\
\hline 30 & Foa & 500 & $\begin{array}{c}\text { New } \\
\text { Zealand }\end{array}$ & $\begin{array}{l}\text { Mucoid discharge, low body } \\
\text { weight and ruffed fur }\end{array}$ & $\begin{array}{l}\text { Sinusitis, trachitis and bilateral } \\
\text { pneumonia }\end{array}$ & 5 & 1 \\
\hline 31 & El-Reyad & 500 & $\begin{array}{c}\text { New } \\
\text { Zealand }\end{array}$ & $\begin{array}{|ll|}\begin{array}{l}\text { Sneezing, cough and nasal } \\
\text { discharge }\end{array} & \\
\end{array}$ & $\begin{array}{lll}\begin{array}{l}\text { Sinusitis, } \\
\text { hydrothorax }\end{array} & \text { trachitis } & \text { and } \\
\end{array}$ & 2 & 0.4 \\
\hline 32 & Kafrelsheikh & 900 & $\begin{array}{c}\text { New } \\
\text { Zealand }\end{array}$ & $\begin{array}{l}\text { Rales, sneezing and mucoid } \\
\text { discharge }\end{array}$ & $\begin{array}{l}\text { Sinusitis, trachitis and unilateral } \\
\text { pneumonia }\end{array}$ & 3 & 0.3 \\
\hline 33 & Dosoque & 600 & Hybrid & $\begin{array}{l}\text { Sneezing, cough, conjunctivitis } \\
\text { and nasal discharge }\end{array}$ & $\begin{array}{l}\text { Conjunctivitis, trachitis and } \\
\text { pneumonia }\end{array}$ & 2 & 0.3 \\
\hline 34 & Qelin & 600 & Hybrid & $\begin{array}{|lc|}\begin{array}{l}\text { Nasal discharge, } \\
\text { discharge and sneezing }\end{array} & \text { ocular } \\
\end{array}$ & $\begin{array}{l}\text { Conjunctivitis, sinusitis and } \\
\text { trachitis }\end{array}$ & 1 & 0.1 \\
\hline 35 & Biala & 1000 & Bosuchate & \begin{tabular}{|l}
$\begin{array}{l}\text { Mild respiratory signs and } \\
\text { sneezing }\end{array}$ \\
\end{tabular} & Sinusitis and trachitis & 2 & 0.2 \\
\hline 36 & Motobes & 600 & Hybrid & $\begin{array}{l}\text { Dull, depression, decreased } \\
\text { body weight and sneezing }\end{array}$ & Sinusitis and trachitis & 2 & 0.3 \\
\hline
\end{tabular}




\begin{tabular}{|c|c|c|c|c|c|c|c|}
\hline 37 & Sakha & 1000 & Hybrid & $\begin{array}{l}\text { Decreased body weight, cough } \\
\text { and sneezing }\end{array}$ & $\begin{array}{l}\text { Trachitis, unilateral pneumonia } \\
\text { and liver congesion }\end{array}$ & 1 & 0.1 \\
\hline 38 & Foa & 500 & $\begin{array}{c}\text { New } \\
\text { Zealand }\end{array}$ & $\begin{array}{l}\text { Depression, ruff fure, in } \\
\text { appetence, sneezing and } \\
\text { mucoid discharge }\end{array}$ & Trachitis, unilateral pneumonia & 2 & 0.4 \\
\hline 39 & Kafrelsheikh & 500 & $\begin{array}{c}\text { New } \\
\text { Zealand }\end{array}$ & $\begin{array}{l}\begin{array}{l}\text { Depression, emaciation and } \\
\text { mild respiratory signs }\end{array} \\
\end{array}$ & $\begin{array}{lll}\begin{array}{l}\text { Sinusitis, } \\
\text { pneumonia }\end{array} & \text { trachitis and } \\
\end{array}$ & 1 & 0.2 \\
\hline 40 & El-Rhyad & 300 & $\begin{array}{c}\text { New } \\
\text { Zealand }\end{array}$ & Dull, depression and sneezing & Trachitis and pneumonia & 2 & 0.6 \\
\hline
\end{tabular}

\section{RESULT AND DISCUSSION}

Twenty one isolates suspected to be ORT were isolated from 300 diseased rabbits with $7 \%$ isolation rate. Examined rabbits were suffered from mild respiratory signs with nasal discharge, depression, ruffled fur, decreased food intake with poor performance, marked dyspnoea and expectoration of blood stained mucous just prior to death. The mortality rate was 0.5 - 5\% (Table 2). Similar findings were obtained by Van Emple et al., (1999).

Table (2): Mortality rate of examined rabbits suffered from respiratory signs

\begin{tabular}{|c|c|c|c|c|c|}
\hline Farm No. & Mortality \% & $\begin{array}{l}\text { Result of } \\
\text { isolation }\end{array}$ & Farm No. & Mortality \% & $\begin{array}{l}\text { Result of } \\
\text { isolation }\end{array}$ \\
\hline 1 & $6.6 \%$ & -ve & 14 & $2 \%$ & -ve \\
\hline 2 & $0.5 \%$ & -ve & 15 & 0.5 & $+\mathrm{ve}$ \\
\hline 3 & $1 \%$ & $+\mathrm{ve}$ & 16 & $2 \%$ & -ve \\
\hline 4 & $0.6 \%$ & $+\mathrm{ve}$ & 17 & $3.3 \%$ & $+\mathrm{ve}$ \\
\hline 5 & $0.4 \%$ & -ve & 18 & $2 \%$ & -ve \\
\hline 6 & $3.3 \%$ & -ve & 19 & $0.6 \%$ & -ve \\
\hline 7 & 1.6 & -ve & 20 & $1.25 \%$ & -ve \\
\hline 8 & $1 \%$ & -ve & 21 & $0.5 \%$ & -ve \\
\hline 9 & $0.6 \%$ & -ve & 22 & $5 \%$ & -ve \\
\hline 10 & $0.6 \%$ & -ve & 23 & $5 \%$ & -ve \\
\hline 11 & $0.75 \%$ & $+\mathrm{ve}$ & 24 & $0.7 \%$ & -ve \\
\hline 12 & $0.5 \%$ & -ve & 25 & $0.6 \%$ & $+\mathrm{ve}$ \\
\hline 13 & $1 \%$ & -ve & 26 & $0.6 \%$ & -ve \\
\hline 27 & $2 \%$ & -ve & 34 & $1.6 \%$ & -ve \\
\hline 28 & $5 \%$ & $+\mathrm{ve}$ & 35 & $0.2 \%$ & -ve \\
\hline 29 & $1 \%$ & -ve & 36 & $0.3 \%$ & -ve \\
\hline 30 & $1 \%$ & $+\mathrm{ve}$ & 37 & $0.4 \%$ & -ve \\
\hline 31 & $0.4 \%$ & -ve & 38 & $0.1 \%$ & -ve \\
\hline 32 & $0.3 \%$ & -ve & 39 & $0.2 \%$ & $+\mathrm{ve}$ \\
\hline 33 & $0.3 \%$ & -ve & 40 & $0.6 \%$ & -ve \\
\hline
\end{tabular}


The main gross lesions in freshly dead and sacrificed rabbits were mild conjunctivitis, mild trachitis, unilateral or bilateral pneumonia with fibrinous exudate in the pleura, pericarditis, peritonitis and petechial haemorrhage on the heart Figs. (1 and 2). Similar findings were reported by El-Gohary and Awaad (1998).

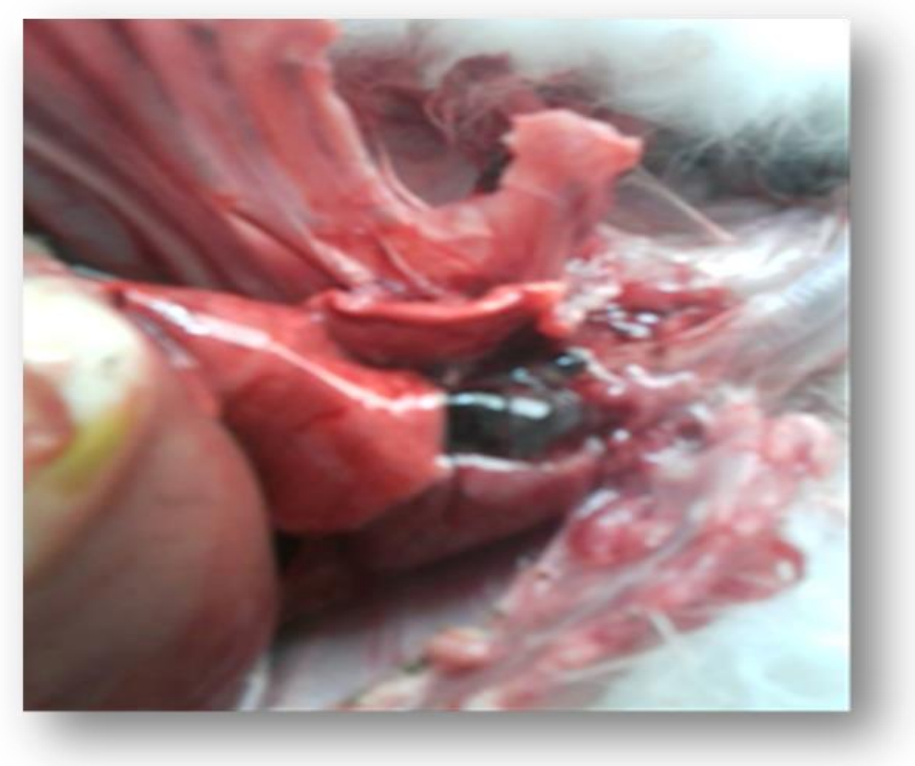

Fig. (1): Pneumonia with fibrinous exudate in the pleura 


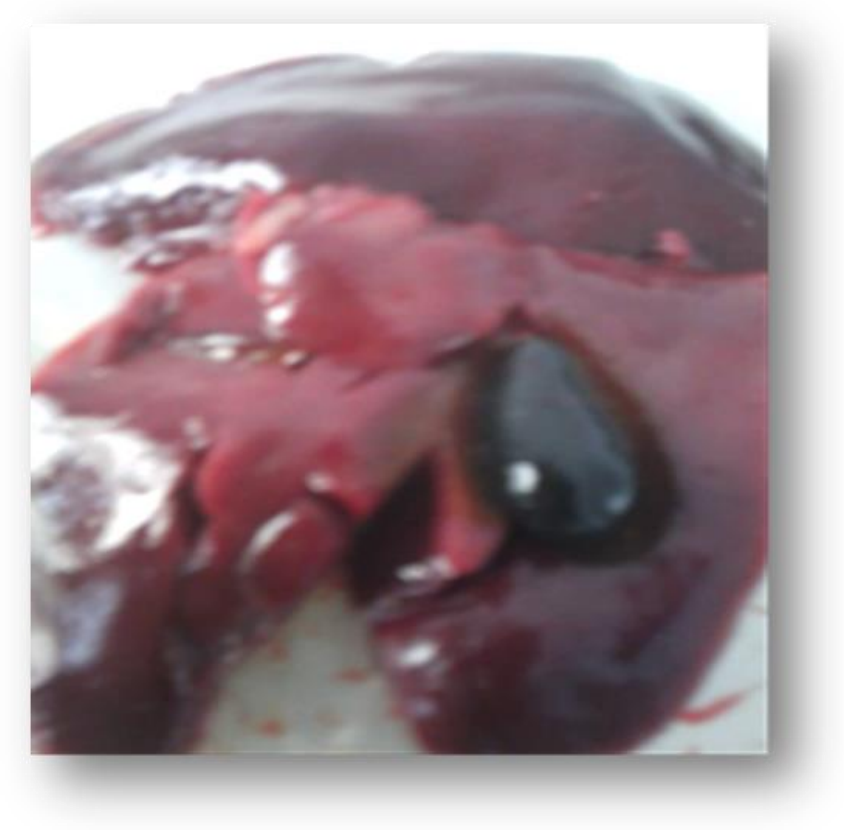

Fig. (2): Pericarditis, peritonitis and haemorrhages on the heart

The incidance of ORT isolation from various organs of examined freshly dead and sacrificed rabbits was described in Table (3). The isolation rate was $7 \%$ (21 out of 300) from sinus, trachea and lungs, $1 \%$ (3 out of 300) from liver and 0\% from heart blood. Similar results were obtained by $\boldsymbol{E l}$ - Abasy (2008) when isolated ORT from internal organs of Muscovy and Balady ducks.

Table (3): Prevalance of ORT isolates from various organs of examined rabbits

\begin{tabular}{|c|c|c|c|c|}
\hline Serial No. & Organs & Examined No. & Positive No. & Isolation rate \\
\hline 1 & Sinus & 300 & 21 & $7 \%$ \\
\hline 2 & Trachea & 300 & 21 & $7 \%$ \\
\hline 3 & Lung & 300 & 21 & $7 \%$ \\
\hline
\end{tabular}

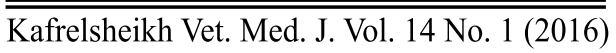




\begin{tabular}{|c|c|c|c|c|}
\hline 4 & Liver & 300 & 3 & $1 \%$ \\
\hline 5 & Heart blood & 300 & 0 & $0 \%$ \\
\hline
\end{tabular}

Colonies of ORT isolated from sinus, trachea, and liver on sheep blood agar media after $24 \mathrm{hrs}$ incubation at $37^{\circ} \mathrm{C}$ appeared as pin point but after $48 \mathrm{hrs}$ incubation appeared as pin head circular, opaque, nonhaemolytic, grey to greyish white, non-pigmented and convex with smooth surface and entire edges. The colonies have characteristic butyric acid odour.

The bacteria appeared as polymorphic, Gram negative, noncapsulated rods after microscopical examination of Gram's stained film prepared from brain heart infusion broth culture after $24 \mathrm{hrs}$ incubation Fig. (3), or on sheep blood agar media Fig. (4). Similar findings were obtained by El-Abasy, (2008) and Sahar, (2011).

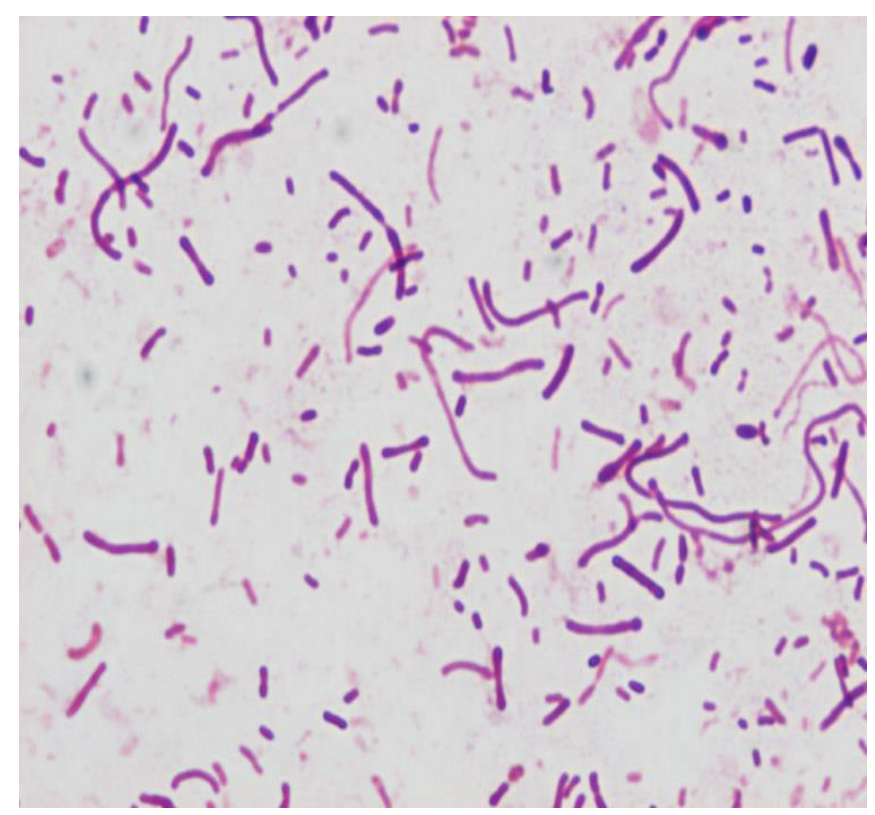

$\overline{\overline{\text { Kafrelsheikh Vet. Med. J. Vol. } 14 \text { No. } 1 \text { (2016) }}}$ 
Fig. (3): Gram negative bacteria isolated on brain heart infusion broth

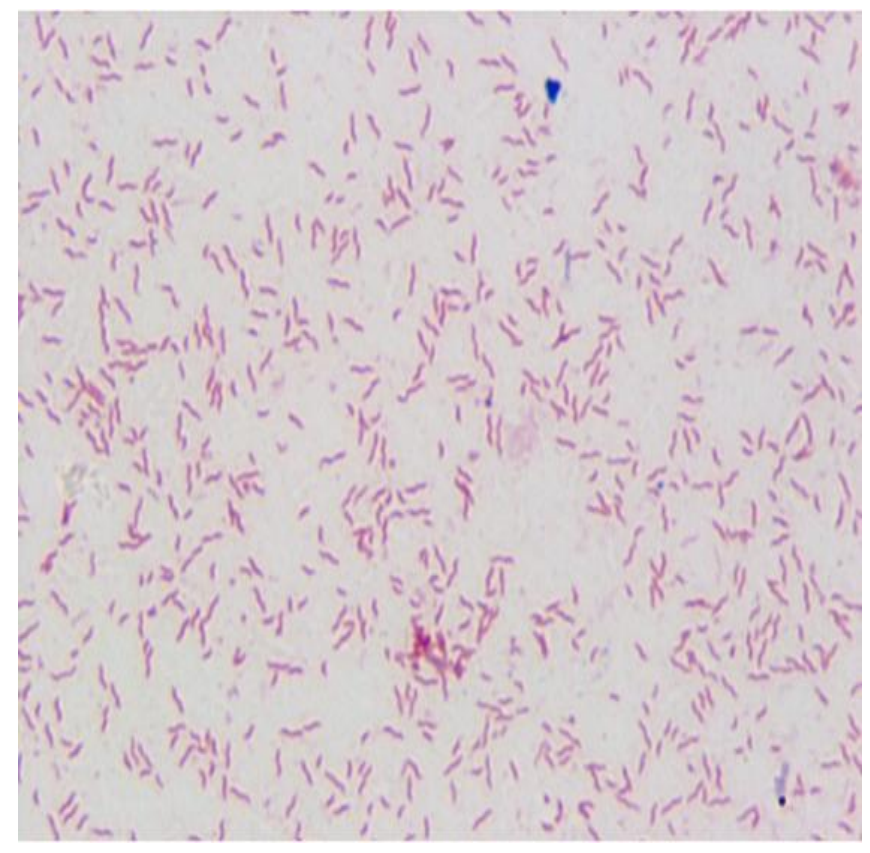

Fig. (4): Gram negative bacteria isolated on sheep blood agar

Biochemical identification of the isolated organisms using API 20 NE strip micro test system revealed positive oxidase, $\beta$-glucosidase, arginine dehydrate, urease and hydrolysis of $\beta$-glucosidase while negative catalase, citrate utilization and. fermentation of glucose, mannitol and arabinose was positive with acid production, while mannose, maltose, carbolic acid and phenile acetic acid were negative. Similar results were obtained by Sahar, (2011).

Table (4): The biochemical activity of suspected ORT isolates with API 20 NE

\begin{tabular}{||c||c||c||c||c|c||c||c|c||c||c|c||c||c||c||c||c||c||c||c||c||c||}
\hline Serial & 1 & $\mathbf{2}$ & $\mathbf{3}$ & $\mathbf{4}$ & $\mathbf{5}$ & $\mathbf{6}$ & $\mathbf{7}$ & $\mathbf{8}$ & $\mathbf{9}$ & $\mathbf{1 0}$ & $\mathbf{1 1}$ & $\mathbf{1 2}$ & $\mathbf{1 3}$ & $\mathbf{1 4}$ & $\mathbf{1 5}$ & $\mathbf{1 6}$ & $\mathbf{1 7}$ & $\mathbf{1 8}$ & $\mathbf{1 9}$ & $\mathbf{2 0}$ & $\mathbf{2 1}$ \\
\hline \hline $\mathrm{NO} 3$ & - & - & - & - & - & - & - & - & - & - & - & - & - & - & - & - & - & - & - & - & - \\
\hline $\mathrm{TRP}$ & - & - & - & - & - & - & - & - & - & - & - & - & - & - & - & - & - & - & - & - & - \\
\hline $\mathrm{GLU}$ & - & - & - & - & - & - & - & - & - & - & - & - & - & - & - & - & - & - & - & - & - \\
\hline $\mathrm{ADH}$ & + & + & + & + & + & + & + & + & + & + & + & + & + & + & + & + & + & + & + & + & + \\
\hline
\end{tabular}




\begin{tabular}{||c||c||c||c||c||c||c||c||c||c||c||c||c||c||c||c||c||c||c||c||c||c||}
\hline \hline URE & + & + & + & + & + & + & + & + & + & + & + & + & + & + & + & + & + & + & + & + & + \\
\hline ESC & + & + & + & + & + & + & + & + & + & + & + & + & + & + & + & + & + & + & + & + & + \\
\hline GEL & - & - & - & - & - & - & - & - & - & - & - & - & - & - & - & - & - & - & - & - & - \\
\hline PNPG & + & + & + & + & + & + & + & + & + & + & + & + & + & + & + & + & + & + & + & + & + \\
\hline GLU & + & + & + & + & + & + & + & + & + & + & + & + & + & + & + & + & + & + & + & + & + \\
\hline ARA & + & + & + & + & + & + & + & + & + & + & + & + & + & + & + & + & + & + & + & + & + \\
\hline MNE & - & - & - & - & - & - & - & - & - & - & - & - & - & - & - & - & - & - & - & - & - \\
\hline MAN & - & - & - & - & - & - & - & - & - & - & - & - & - & - & - & - & - & - & - & - & - \\
\hline NAG & - & - & - & - & - & - & - & - & - & - & - & - & - & - & - & - & - & - & - & - & - \\
\hline MAL & - & - & - & - & - & - & - & - & - & - & - & - & - & - & - & - & - & - & - & - & - \\
\hline GNT & - & - & - & - & - & - & - & - & - & - & - & - & - & - & - & - & - & - & - & - & - \\
\hline CAP & - & - & - & - & - & - & - & - & - & - & - & - & - & - & - & - & - & - & - & - & - \\
\hline ADI & - & - & - & - & - & - & - & - & - & - & - & - & - & - & - & - & - & - & - & - & - \\
\hline MLT & - & - & - & - & - & - & - & - & - & - & - & - & - & - & - & - & - & - & - & - & - \\
\hline CIT & - & - & - & - & - & - & - & - & - & - & - & - & - & - & - & - & - & - & - & - & - \\
\hline PAC & - & - & - & - & - & - & - & - & - & - & - & - & - & - & - & - & - & - & - & - & - \\
\hline OX & + & + & + & + & + & + & + & + & + & + & + & + & + & + & + & + & + & + & + & + & + \\
\hline
\end{tabular}

$\mathrm{NO}_{3}$ : Potassium nitrate reduction of nitrates to nitrites.

GLU: D- glucose fermentation (Glucose).

ADH: L-arginine Arginine D-hydrolase.

GEL: Gelatine hydrolysis (protease).

GLU: D-glucose assimilation (Glucose).

MNE: D-mannose assimilation (Mannose).

MAN: D-mannitol assimilation

MAL: D-maltose assimilation (maltose).

CAP: Capric acid assimilation (capric acid)
TRP: L-Tryptophane indole production.

ESC: esculin ferric citrate -hydrolysis (B-glucosidase)

URE: Urea urease.

PNPG: 4.nitro phenyl- $\beta \mathrm{D}$-galactopyranoside- $\beta$-galactosidase.

ARA: L-arabinose assimilation (arabinose)

CIT: Trisodium citrate assimilation

NAG: $\mathrm{N}$-acetyl glucose amine-assimilation Glucosamine.

GNT: Potassium gluconate-assimilation

ADI: Adipic acid assimilation (Malate)

Serological identification of suspected ORT isolates which were subjected to serotyping using specific antisera against ORT (A-R) by AGPT revealed that most of isolates were belonged to Serotype A. (Table 5) similar results were obtained by Attia, (2008).

Table (5): Serotyping of suspected ORT strains by agar gel precipitation test

\begin{tabular}{|c|c|c|c|}
\hline Serial No. & Date & Organ & ORT serotype \\
\hline 1 & $11 / 5 / 2014$ & Lung & $\mathrm{A}$ \\
\hline 2 & $11 / 5 / 2014$ & Lung & $\mathrm{A}$ \\
\hline 3 & $11 / 5 / 2014$ & Lung & A \\
\hline 4 & $11 / 5 / 2014$ & Lung & A \\
\hline 5 & $11 / 5 / 2014$ & Trachea & $\mathrm{A}$ \\
\hline 6 & $11 / 5 / 2014$ & Trachea & $\mathrm{A}$ \\
\hline 7 & $11 / 5 / 2014$ & Trachea & Untypable \\
\hline
\end{tabular}

Kafrelsheikh Vet. Med. J. Vol. 14 No. 1 (2016) 


\begin{tabular}{|c|c|c|c|}
\hline 8 & $11 / 5 / 2014$ & Trachea & untypable \\
\hline 9 & $11 / 5 / 2014$ & Sinus & A \\
\hline 10 & $18 / 5 / 2014$ & Sinus & A \\
\hline 11 & $18 / 5 / 2014$ & Sinus & A \\
\hline 12 & $18 / 5 / 2014$ & Sinus & Untypable \\
\hline 13 & $18 / 5 / 2014$ & Sinus & A \\
\hline 14 & $18 / 5 / 2014$ & Sinus & A \\
\hline 15 & $18 / 5 / 2014$ & Sinus & A \\
\hline 16 & $18 / 5 / 2014$ & Lung & A \\
\hline 17 & $18 / 5 / 2014$ & Lung & A \\
\hline 18 & $18 / 5 / 2014$ & Lung & A \\
\hline 19 & $18 / 5 / 2014$ & Trachea & A \\
\hline 20 & $18 / 5 / 2014$ & Trachea & A \\
\hline 21 & $18 / 5 / 2014$ & Trachea & A \\
\hline
\end{tabular}

The antibiogramme of ORT isolated strains was investigated against 19 antimicrobial agents using disc diffusion technique revealed that ORT isolates were sensitive to sulphamethxazol-trimethoprin, , oxytetracyclin, enrofloxacin, ciprofloxacin, amoxycillin, ampicillin, tetracycline, erythromycin and sipramycine but resistance to penicillin, streptomycin, clindamycin, colistine sulphate, lincomycine, gentamycin, vancomycin and neomycin (Table 6). Similar results were reported by Devriese et al., (2001).

Table (6): Results of in vitro sensitivity test of ORT isolated strains against 19 antimicrobial agents

\begin{tabular}{|c|c|c|c|c|c|}
\hline \begin{tabular}{|c|} 
Serial \\
No.
\end{tabular} & Antibacterial agent & $\begin{array}{c}\text { Potency of disc } \\
(\mu \mathrm{g})\end{array}$ & \begin{tabular}{|c} 
Standard sensitivity \\
zone $(\mathrm{mm})$
\end{tabular} & $\begin{array}{c}\text { Zone of inhibition } \\
(\mathrm{mm})\end{array}$ & $\mathbf{S} / \mathbf{R}$ \\
\hline 1 & Oxytetracyclin & 30 & $14-19$ & 14 & $\mathrm{~S}$ \\
\hline 2 & Enrofloxacin & 5 & $10-15$ & 10 & $\mathrm{~S}$ \\
\hline 3 & Penicillin $\mathrm{G}$ & 10 & - & - & $\mathrm{R}$ \\
\hline 4 & Ciprofloxacin & 5 & $15-21$ & 18 & $S$ \\
\hline 5 & Streptomycin & 10 & $17-22$ & 16 & $\mathrm{R}$ \\
\hline 6 & Clindamycin & 2 & - & - & $\mathrm{R}$ \\
\hline 7 & Amoxicillin+clavulanic acid & 30 & - & 10 & $\mathrm{~S}$ \\
\hline 8 & Amoxicillin & 25 & $23-31$ & 27 & $\mathrm{~S}$ \\
\hline 9 & Ampicillin & 10 & $13-17$ & 15 & $\mathrm{~S}$ \\
\hline
\end{tabular}

Kafrelsheikh Vet. Med. J. Vol. 14 No. 1 (2016) 
Ornithobacterium Rhinotracheale (ORT) Infection ...

Moshira, A. El-Abasy et., al.

\begin{tabular}{||l||c||c||c||c||}
\hline \hline 10 & Nitrofurantoin & 300 & - & - \\
\hline 11 & Colisten sulphate & 25 & $13-18$ & 0 \\
\hline 12 & Tetracyclin & 10 & $14-20$ & 17 \\
\hline 13 & Lincomycin & 2 & - & 0 \\
\hline 14 & Gentamycin & 10 & $12-15$ & 0 \\
\hline 15 & Vancomycin & 30 & $10-18$ & 0 \\
\hline 16 & Erythromycin & 30 & $14-19$ & $\mathrm{R}$ \\
\hline 17 & Neomycin & 30 & - & 14 \\
\hline 18 & Spiramycin & 100 & - & 11 \\
\hline 19 & Sulphamethoxazole+trimethopriem & 25 & $10-16$ & $\mathrm{R}$ \\
\hline
\end{tabular}

Depression, decreased feed intake, ruffled fur and sneezing with transient nasal discharge were observed in experimentally infected 3month-old rabbits. Mortality rate was $13 \%$ (Table 7). Post mortem lesions in dead and sacrificed rabbits were trachitis with tracheal exudate, unilateral and bilateral pneumonia with lung congestion, pleuritis, pericarditis, enlarged and congestion liver and spleen. The organism was re-isolated from trachea and lungs. These results are similar to those reported by Hafez, (1996) El-Gohary and Awaad, (1998) and El-Abasy. (2008).

Table (7): Lesion score and mortality of ORT experimentally infected rabbits

\begin{tabular}{||c||c||c||c||c||c||c||c||}
\hline \multirow{2}{*}{$\begin{array}{c}\text { Group } \\
\text { No. }\end{array}$} & \multicolumn{9}{|c||}{ Lesions scores } & Mortality \\
No.
\end{tabular}

$1 \mathrm{~A}=$ infected with ORT and untreated. $2 \mathrm{~A}=$ rabbits infected with ORT and treated with sulpha-.trimethoprim Kafrelsheikh Vet. Med. J. Vol. 14 No. 1 (2016) 


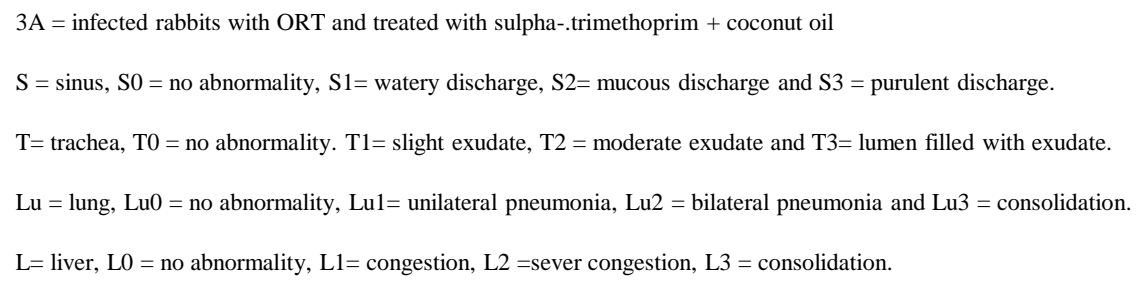

Treatment trials were based on the results in vitro antibiogramme of the isolated ORT organisms. The clinical signs and post-mortem lesions were disappeared in infected rabbits and treated with sulphatrimethoprine in drinking water for 5 consecutive days with dietary supplementation with coconut oil when compared with infected untreated control group. These results were similar to that reported by Shihata and Ibrahim, (2004) and El-Abasy, (2008).

Histopathological examination of lungs in ORT infected untreated rabbits revealed fibrinous pneumonia, emphysema and haemorrhage (Fig 5). Lungs of ORT infected rabbits and treated with sulpha-trimethoprim plus coconut oil showed marked improvement as shown in (Fig. 6) while control group shown no pathognomonic lesions (Fig.7). 
Moshira, A. El-Abasy et., al.

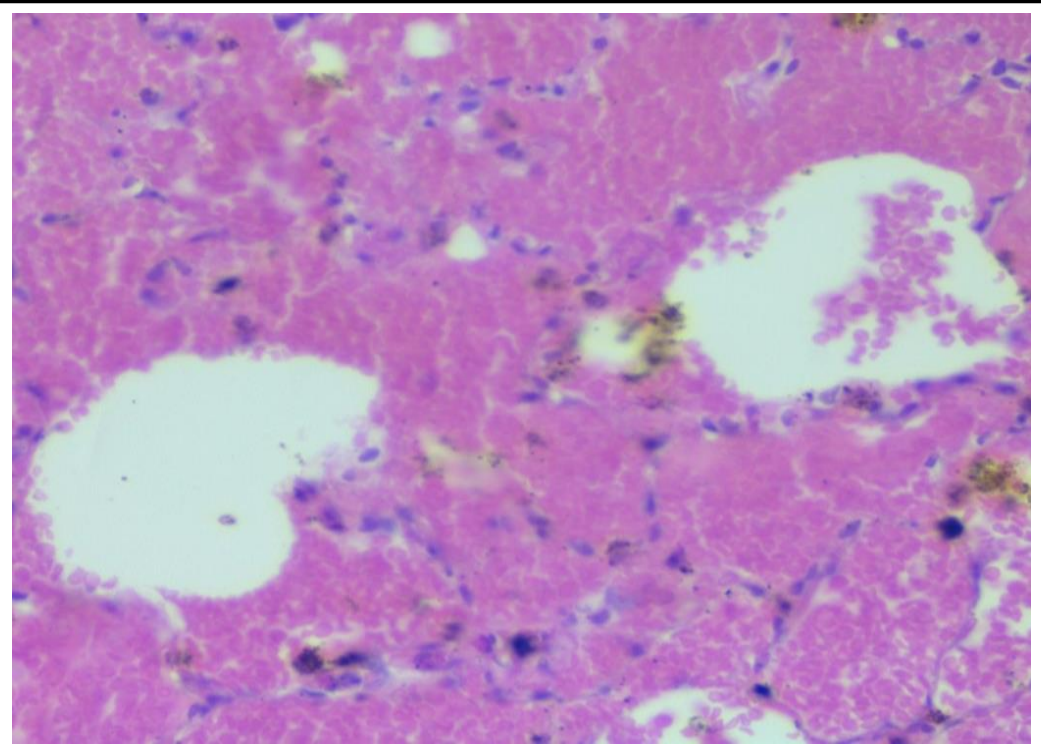

Fig. (5): lung of ORT infected untreated rabbits showed fibrinous pneumonia, emphysema and haemorrhage.

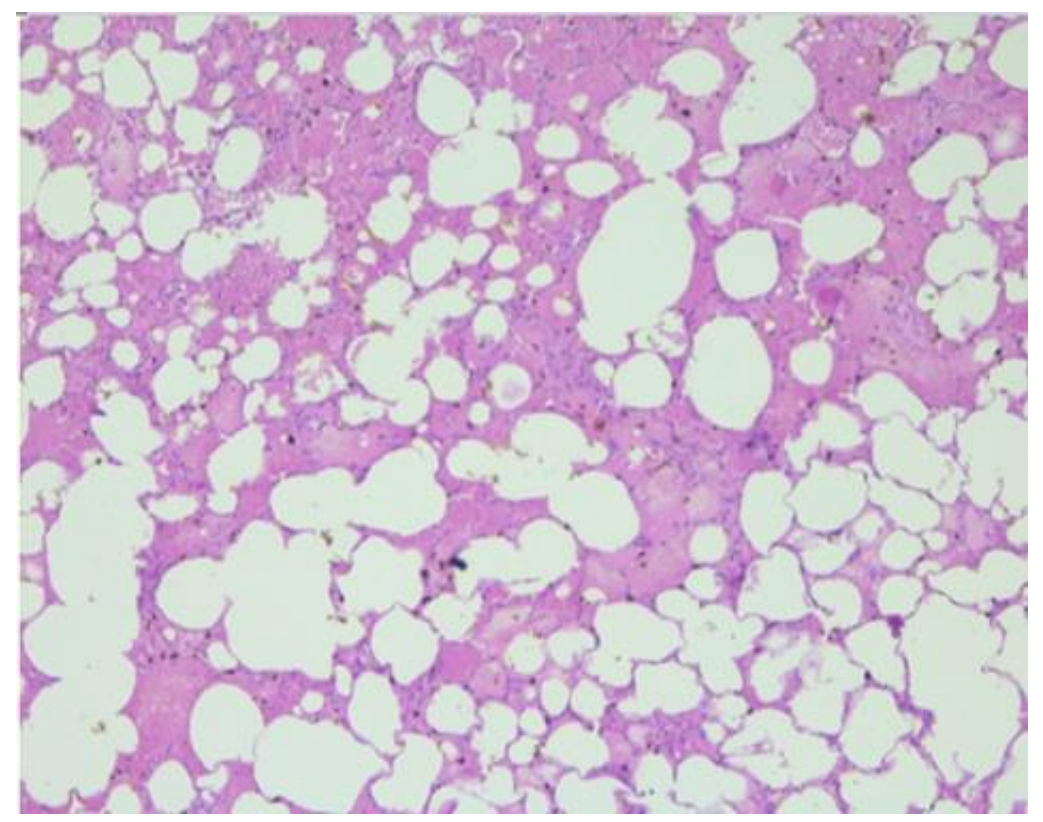

Fig. (6): lung of ORT infected rabbits and treated showed mild fibrinous pneumonia and mild congestion 
Microscopical examination of liver of ORT infected untreated rabbits revealed severe lesions as thrombus, haemorrhage and inflammatory cell infiltration Fig (7). Sulpha--trimethoprim + coconut oil treatment of infected rabbits revealed improvement in liver lesions Fig (8).

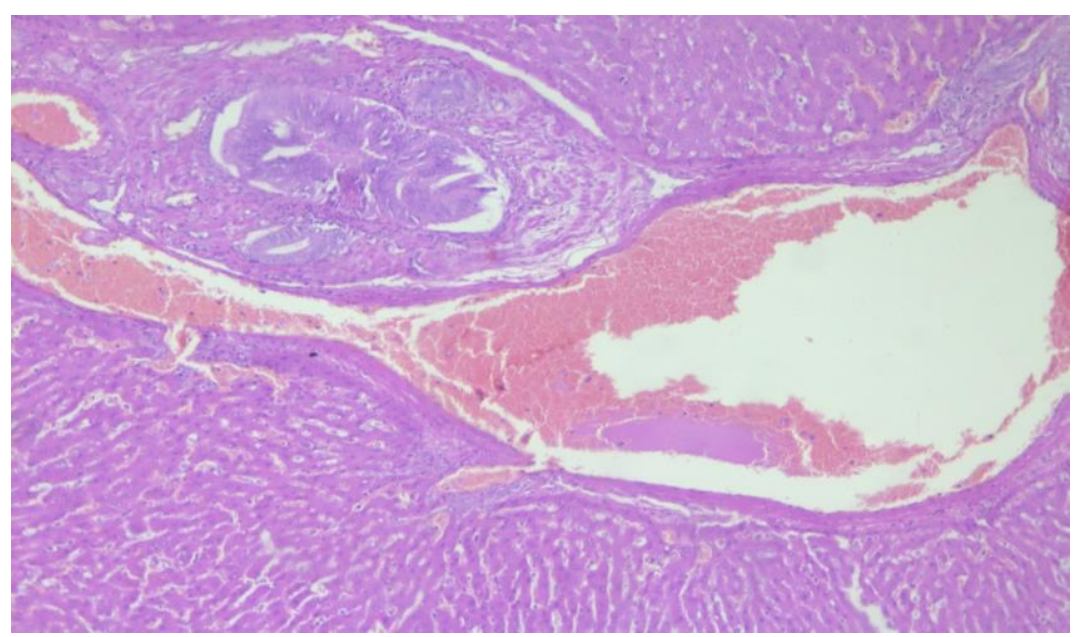

Fig. (7): liver of infected untreated rabbits showing thrombus, haemorrhage and inflammatory cells infiltration.

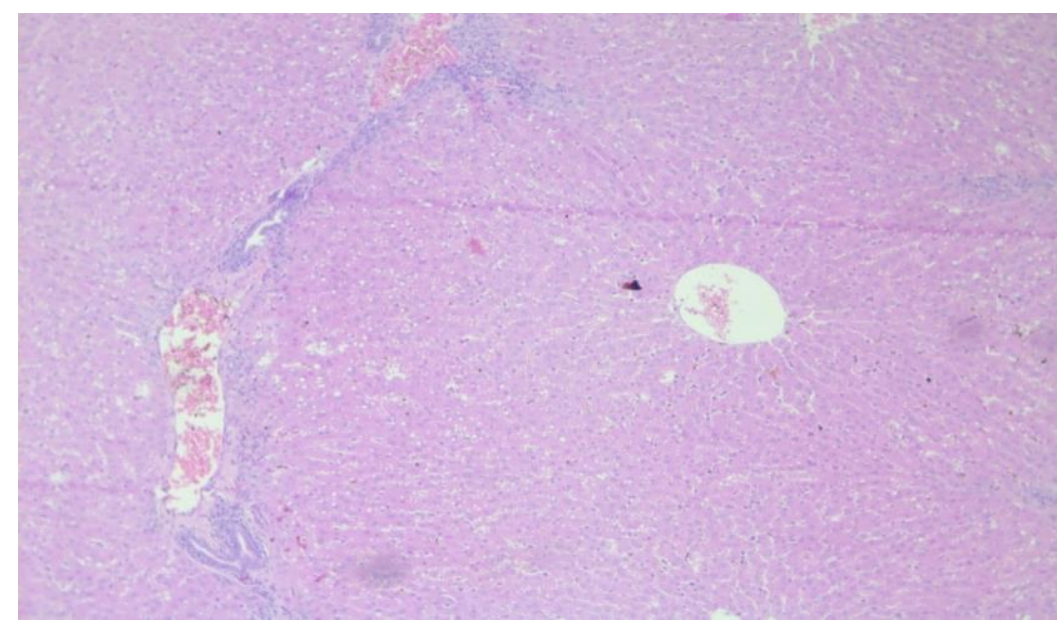

Fig. (8): Liver of infected and treated rabbits showing mild congestion and mild inflammatory cells infiltration. 


\section{CONCLUSION}

ORT is claimed to be a new pathogen isolated from diseased rabbits in Kafrelshikh Governorate, Egypt. Further studies on the pathogenicity of single and mixed infection and trials for production of potent vaccine for protection of rabbits against ORT infection are needed.

\section{REFERENCES}

- Attia, S. A., (2008): Studies on Ornithobacterium rhinotracheale infection in rabbits. M.V.SC. Thesis.

- Back, A.; Nagaraja, K. V and Halvorson, D. (1996): Preiminary studies on Ornithobacterium rhinotracheale. In Proceedings of the turkey symposium, September 4-6, Minneapolis, Minnesota,PP:29-31.

- Back, A.; Staphanie, S.; Rajaskears, G. and Halvorson, D. (1997): ORT isolation from different geographic location $.48^{\text {th }}$ North Central Avian Disease Conference: 22-24.

- Bancroft, J. D. and Gamble, M., (2007): Theory and Practice of Histological Techniques $.5^{\text {th }}$ Ed. Churchill Livingston, London, UK, PP: 125-138.

- Chalton, B.; Channing; S.; Santiagio, S.; Bickford, A.; ordona, C.; Chin, R.; Cooper, G.; Droual, R.; Jeffer, J.; Meteyer, C.; Shivaprassad,H. and Walker, R.(1993): Preliminary characterization of pleomorphic Gram negative rod associated with avian respiratory disease. J of Veterinary Diagnostic Investigation, 5: 47-51.

- Chin, P. R. and R. B. Chalton (2000): Ornithobacteriosis Avian pathologist $4^{\text {th }}$ Ed. of American Veterinary Association. PP: 89-91.

- Devriese, L. A. , De Herdt., P. and Haesebrouck, (2001): Antibiotic sensitivity and resistance in Ornithobacterium rhinotracheale strains from Belgian broiler chickens. Avian Pathology, 30: 197-200. 
- El-Abasy, M. A., (2008): Ornithobacterium rhinotracheale (ORT) in Muscovy and Balady ducks. Proc. $8^{\text {th }}$ Sci. Conf. Egypt. Vet. Poult. Assoc. PP: 133- 139.

- El-Gohary, A.A. and M. H.H. Awaad (1998): Concomittant Ornithobacterium rhinotracheale(ORT) and E.Coli infection in chicken broilers.Veterinary Medicine Journal of Giza 45:67-75.

- El-Gohary, A. A., (1998): Ornihobacterium rhinotracheale (ORT) associated with hatching problems in chicken and turkey eggs. Veterinary Medicine Journal of Giza, 46: 183- 191

- Hafez, H. M. (1996): Current status on the role of Ornithobacterium rhinotracheale (ORT) in respiratory disease complexes in poultry. Archiev. Fur Gelfugelkunde, 61: 208-211.

- Lillie, R.D. (1984): Histopathological techniques, $3^{\text {rd }}$ Ed. The Blankiston Company, Philadelphia.

- Petri and Waston, (1999): Staistics for Veterinary and Animal Science, $1^{\text {th }}$ Ed., U.S.A. Prevalence of antibiotics against ORT in broilers and breeders in Southern Brazil. Avian Diseases, 47: 731-737.

- Roussan, D. A.; Al-Rifai, R.H.; Khawldeh, G.Y.; Totanji, W.S. and Shaheen, I. (2011): Ornithobacterium rhinotracheale and Mycoplasma synoviae in broiler chickens in Jordan Rev. Sci.of Int. Epiz. 30: 931 - 937.

- Shihata, A. B and Ibraheem, O. B., (2004): Ornithobacterium rhinotracheale (ORT) in some birds and rabbits at Sharkia Governorate.Proc. $6^{\text {th }}$ Sci.Conf. Egypt. Vet. Poult.Assoc. PP: 288-298.

- Sahar, S. A., (2011): Conventional and molecular identification of Ornithobacterium rhinotracheale isolated from turkey.Ph. D Thesis.

- Van Emple, P. C.; Vrijen hoek, M., M..; Goovaerts, D. and Vanden B., (1999): Immunohistochemical and serological investigation of experimental Ornithobacterium rhinotracheale infection in chickens. Avian Pathology. 28: 187-193. 